\title{
Clinical Outcomes of Modified versus Traditional Technique for the Surgery of Hydatid Cyst of the Liver: A Case Control Study
}

\author{
Asieh Sadat Fattahi ${ }^{1}$, Seyed Hossein Fattahi Masoom ${ }^{2, *}$, Farjad Lorestani ${ }^{3}$, Mehrdad Fakhlai ${ }^{4}$, \\ Fatemeh Sadat Abtahi Mehrjerdi ${ }^{4}$, Mehrdad Gazanchian ${ }^{5}$, Golshid Nouri Hosseini ${ }^{5}$
}

1. Associate Professor of General Surgery, Endoscopic and Minimally Invasive Surgery Research Center, Mashhad University of Medical Sciences, Mashhad, Iran

2. Professor of Thoracic Surgery, Lung Diseases Research Center, Mashhad University of Medical Sciences, Mashhad, Iran

3. General Surgery, Endoscopic and Minimally Invasive Surgery Research Center, Mashhad University of Medical Sciences, Mashhad, Iran

4. Resident of General Surgery, Endoscopic and Minimally Invasive Surgery Research Center, Mashhad University of Medical Sciences, Mashhad, Iran

5. School of Medicine, Mashhad University of Medical Sciences, Mashhad, Iran

\section{* Corresponding Author:}

Seyed Hossein Fattahi Masoom, MD Lung Diseases Research Center, Mashhad University of Medical Sciences,

Mashhad, Iran

Telefax: + 985138012841

Email: emis@mums.ac.ir

Received: 23 Dec. 2018

Accepted: 03 May 2019

\section{ABSTRACT}

\section{BACKGROUND}

Echinococcus granulosis is a parasitic infection most commonly involving the liver. Iran is a hyperendemic area for this disease according to WHO. Despite improvements in medical and interventional radiological techniques, surgery remains the gold standard of treatment; however evidence on different surgical modalities were explained. Considering the high population of referring patients presenting to Omid and Ghaem Hospitals, Mashhad, Iran, we decided to compare the complications of our modified technique with routine technique in hydatid cyst surgery.

\section{METHODS}

56 patients with hydatid cyst of the liver who underwent modified and routine surgical treatment in Ghaem and Omid Hospitals Mashhad, Iran were studied during Aug 2013- Nov 2015. 27 patients underwent modified surgical technique, whereas the remaining 27 patients were treated by using routine surgical method. These two groups of patients were compared with each other according to their postoperative length of hospital stay and resulting complications.

\section{RESULTS}

The mean age of our patients was 41 years. 27 patients were male and 29 were female. Our results showed no statistically significant difference regarding the incidence of postoperative complications between the two groups. However, mean length of hospital stay was significantly different between the groups $(4.5 \pm 1.87$ and $7.6 \pm 2.25$ days, respectively, $p<0.001)$.

\section{CONCLUSION}

The method of modified surgery with closed cyst drainage, which does not use external drains, is a safe surgical modality in the treatment of hydatid cyst disease of the liver if applied properly on appropriate patients.

\section{KEYWORDS:}

Hydatid Cyst, Hepatic, Surgical procedure, Digestive system, Postoperative complications

Please cite this paper as:

Sadat Fattahi A, Fattahi Masoom SH, Lorestani F, Fakhlai M, Abtahi Mehrjerdi FS, Gazanchian M, Nouri Hosseini G. Clinical Outcomes of Modified versus Traditional Technique for the Surgery of Hydatid Cyst of the Liver: A Case Control Study. Middle East J Dig Dis 2019;11:152-157. doi: 10.15171/mejdd.2019.142.

\section{INTRODUCTION}

Echinococcus granulosis is a parasitic disease endemic in Mediterranean regions, Middle East, South America, New Zealand, Australia, and China. It is a heavy burden on public health in these countries. Recent epidemiological data showed an increase in the incidence of this disease in central Europe. Iran is reported as a hyperendemic region. The prevalence of human hydatid cyst is between $1.1 \%$ to $13.7 \%$ in various parts of Iran. ${ }^{1}$ 


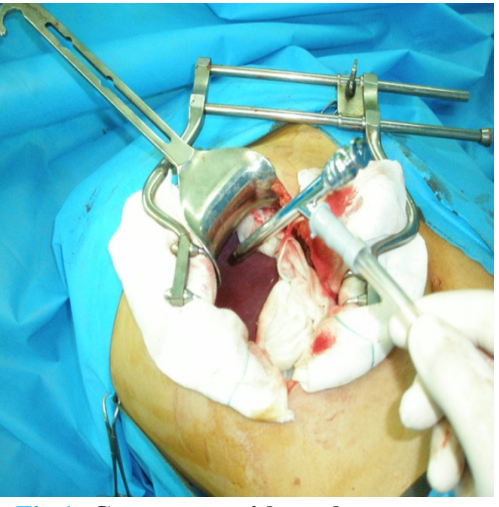

Fig.1: Cystostomy with trochar cyst

The most common involved organ is the liver and most liver cysts are asymptomatic. Patients may present with abdominal pain mainly in epigastric or right subcostal regions, hepatomegaly, ascites, nausea and vomiting, fever, infectious complications, or rupture of the cysts. ${ }^{2}$ Despite recent improvements in non-surgical and interventional radiological techniques in the treatment of hydatid cyst, surgery is still among the first choices. ${ }^{3}$ Medical treatments such as albendazole or mebendazole may not be adequate for the treatment of hydatid cysts, however they are widely used alongside surgery for complete eradication of protoscolices and easier removal of germinal layer during surgery. ${ }^{4}$ Even if one viable scolex remains after surgery, it can multiply and create millions of parasites; therefore scoliocides are used in all surgical or interventional radiological techniques.

Available surgical techniques are liver resection with cystectomy or cystostomy and evacuation of parasites. Newer surgical techniques include laparoscopic techniques that showed promising results; however they have not shown significant superiority over conventional open surgery techniques, thus open surgery is still widely performed. ${ }^{5}$

Goals of surgery are inactivation and evacuation of cysts and preventing the recurrence of the disease. Despite large number of surgeries done on hydatid cysts, few studies are available regarding the post operative complications and recurrence of the disease. Reports showed the recurrence rate to be from $2 \%$ to $25 \%$ based on the size, location, number of cysts, the ability of surgical team, and the rupture of cyst before surgery. ${ }^{6-8}$

Considering the large number of patients presenting to Ghaem and Omid Hospitals, Mashhad, Iran and different surgery methods available, we planned to compare our

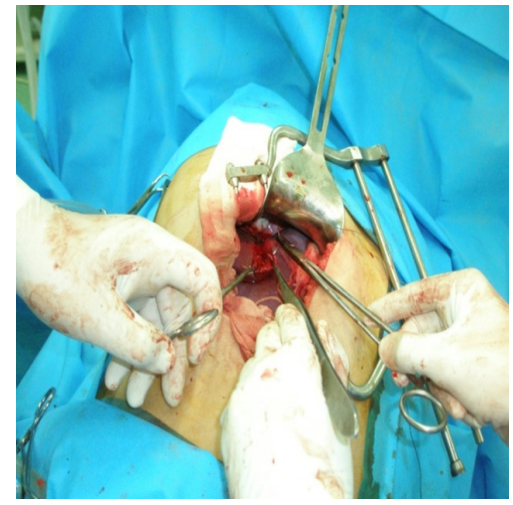

Fig.2: Cyst cavity capitonnage

routine surgical technique with newer less invasive technique for hydatid cysts surgery.

\section{MATERIALS AND METHODS}

We conducted a prospective, case control study on hospital in patients diagnosed as having liver hydatid cyst in Omid and Ghaem Hospitals, Mashhad, Iran. Patients were selected using simple non-randomized sampling. The inclusion criterion was: All patients with one to three liver hydatid cysts who were candidates for surgery.

Exclusion criteria were: Multiple liver cysts (more than three cysts), simultaneous cysts in the liver and another organ, and patients who could not tolerate surgery. According to the aim of the study and other similar studies and considering type one and type two error of $5 \%$ and $20 \%$, respectively, sample size was calculated to be 28 patients in each group using the following formula:

$$
\begin{array}{ll}
n=\frac{\left(z_{1-\frac{\alpha}{2}}+Z_{1-\beta}\right)^{2}\left(P_{1}\left(1-P_{1}\right)+P_{2}\left(1-P_{2}\right)\right)}{(d)^{2}} \\
\mathrm{P}_{1}=0.39 & \mathrm{P}_{2}=0.1 \\
\mathrm{n}_{1}=\mathrm{n}_{2}=28 & \mathrm{~d}=\mathrm{p}_{1}-\mathrm{p}_{2}=0.29 \\
z_{1-\frac{\alpha}{2}}=1.96 & Z_{1-\beta}=0.84
\end{array}
$$

\section{Methods:}

56 patients diagnosed with liver hydatid cysts were categorized into two groups based on the surgical methods. One surgical team exclusively operated on each group. Demographic patients' information was collected using patients' files. The description of two different surgery methods used in our study is as follows (figures 1, 2, and 3): 

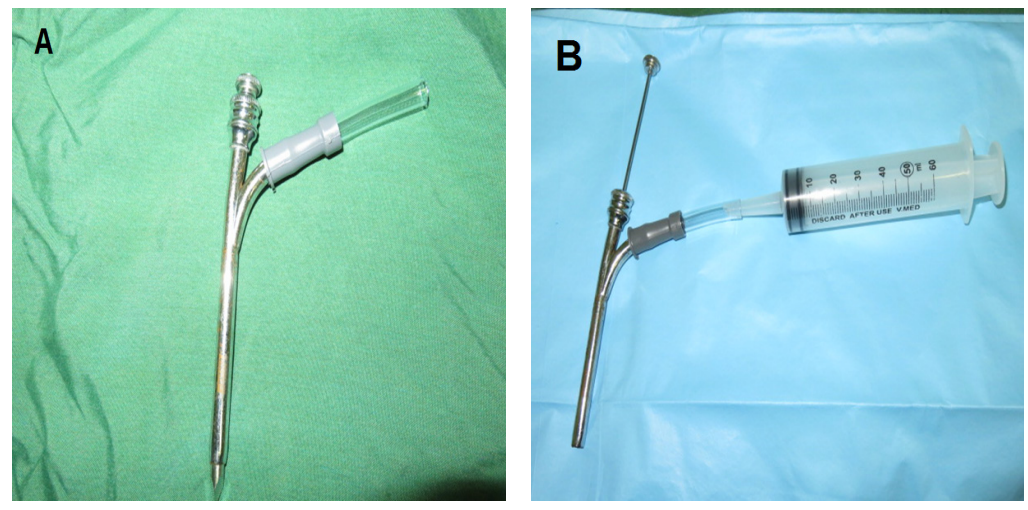

Fig.3: Modified tube for cyst drainage

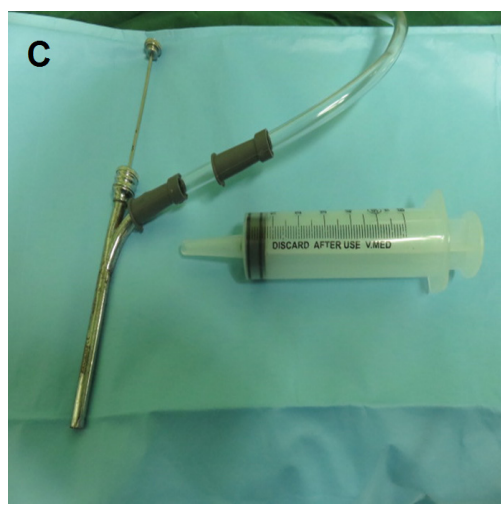

\section{Conventional technique:}

Under general anesthesia and endotracheal intubation in supine position with a roll under right upper quadrant (RUQ) of the abdomen, a Kocher incision was made. After placing moistened gases with hypertonic saline $25 \%$ inside the abdominal cavity and around the liver, cystostomy was done by trochar cyst and cyst fluid was suctioned. After evacuation of the cyst, its wall was opened. Sometimes a piece of germinative layer was left in the cyst; therefore we took it out carefully. The liver wall around the cyst was also resected and capitonnage was done for the cavity by 0 or 1 Vicryl suture. If capitonnage was not possible Petzer drain was put into the cyst and tube cystostomy was done. Moistened gases were taken out and the abdominal cavity was irrigated with normal saline. Then one or two red Nélaton tubes were placed around the liver at the cyst location. The abdominal wall was then repaired in the anatomical plan. The overlying skin was repaired using far and near sutures. If secretions were less than $100 \mathrm{~mL}$ daily and bleeding or biliary secretions stopped, the drains could be removed.

\section{Modified technique:}

Like the routine technique, we used the same position and incision under general anesthesia. But we did a modification for drainage of the cyst. We cut a suction tube about $10 \mathrm{~cm}$ and connected it to another suction, after that the cyst trochar was connected to the suction, then we clamped the first suction tube and detected the other suction and $50 \mathrm{cc}$ hypertonic saline $25 \%$ was injected in to the cyst and the suction was re-attached to the trochar cyst. This cycle was repeated 7-8 times (closed suction drainage). After evacuation of the cyst, its wall was opened and we looked for remaining germinative layer. We used capitonnage for the remaining wall for closure without drain in the abdominal cavity (figure 3). After 3 or 4 days we performed ultrasonography to find fluid and then we discharged the patient if he had no comlication.

The patients were then followed up for their duration of hospital stay and the following variables were recorded for each patient (the length of follow-up was $14 \pm 5$ months) and used for further analysis: Patient's sex, age, cyst location, cyst size, surgery technique, length of hospital stay, post-surgery infection, hematoma, seroma, and secondary intervention.

\section{Statistical analysis:}

Statistical analysis was carried out using SPSS software ver. 17. In order to evaluate the distribution of two qualitative variables $\mathrm{Chi}^{2}$ test was used. For quantitative variables, Kolmogorov Smirnoff test was used to assess the normality. If data were normally distributed, independent $t$ test was used otherwise the equivalent nonparametric test was used. Level of significance was set at $p$ value $<0.05$.

\section{RESULTS}

In a prospective study we evaluated 56 patients with liver hydatid cyst undergoing surgery. The patients were divided into two groups based on the surgery method (routine or modified) each containing 28 patients.

29 patients were male and 27 were female. Mean age 
Table 1: Characteristics of routine and modified surgery groups

\begin{tabular}{|c|c|c|c|c|}
\hline \multicolumn{2}{|l|}{ Variables } & Routine surgery & Modified surgery & $P$ Value \\
\hline \multicolumn{2}{|l|}{ Age(Years old) } & $54 / 12 \pm 2 / 39$ & $82 / 16 \pm 7 / 42$ & $0.377^{\mathrm{a}}$ \\
\hline \multicolumn{2}{|l|}{ Sex $(\%$ Male $)$} & $46 \%$ & $50 \%$ & $0.789^{b}$ \\
\hline \multirow[t]{3}{*}{ Cyst Location } & Right lobe (\%) & $57.1 \%$ & $67.9 \%$ & \multirow[t]{3}{*}{$0.704^{b}$} \\
\hline & Left Lobe (\%) & $25 \%$ & $17.9 \%$ & \\
\hline & Both lobes $(\%)$ & $17.9 \%$ & $14.3 \%$ & \\
\hline \multicolumn{2}{|l|}{ Infection (n) } & 2 & 0 & $0.491^{\mathrm{c}}$ \\
\hline \multicolumn{2}{|l|}{ Hematoma (n) } & 1 & 0 & $>0.99^{c}$ \\
\hline \multicolumn{2}{|l|}{ Seroma (n) } & 1 & 2 & $>0.99^{\mathrm{c}}$ \\
\hline \multicolumn{2}{|c|}{ Length of hospital stay (days) } & $7.6 \pm 2.25$ & $4.5 \pm 1.87$ & $<0.001^{\mathrm{d}}$ \\
\hline
\end{tabular}

a: independent sample t-test; b: chi square; c: Fisher's exact test; d: Mann-Whitney U test

of our subjects was 41 years. Of the 56 patients, 35 had cysts in the right lobe, 12 in the left lobe and 9 patients had cysts in both lobes of the liver. Post surgery complications included two Infections, one hematoma, and three seromas. None of our patients died and we observed no biliary leakage. Length of hospital stay was between 2 to 11 days with a mean of 6 days. 7.6 days in the routine group in comparison with 4.5 days in the modified group. Post surgery complications were more common in routine technique; however no statistical significance was observed. The only statistical difference between the routine and modified surgery groups was the length of hospital stay, which was shorter in modified group compared with the routine group (table 1). It seems that with using drains the surgeon tended to discharge the patients later. The demographic data and cyst characteristics were not significantly different between the two groups. The mean follow-up was $14 \pm 5$ months. Two patients in the modified group had fluid in cyst cavity in follow-up sonography. They underwent percutaneous drainage and recovered without any more drain. We had one case of seroma in the routine group. We had no recurrence during the follow-up period; however maybe more time are needed to evaluate this between the two groups.

\section{DISCUSSION}

Here we reported a prospective case control study comparing 56 patients undergone liver hydatid cyst surgery. We compared the routine versus modified surgery techniques and our results showed that post surgery complications were not statistically different between the two groups; however length of hospital stay was significantly shorter in the modified group, which can lead to less cost.

Surgery alongside medical therapy is the mainstay of treatment for liver hydatid cyst. The main goals of surgery are inactivation and evacuation of cysts and preventing the recurrence. However, ultrasonographic follow-up of liver cysts with a diameter of less than $4 \mathrm{~cm}$, cysts deep within liver parenchyma, or calcified liver cysts are better than operating on them. ${ }^{9}$ Multiple surgery methods have been introduced, but not enough randomized controlled trial have been carried out to suggest the best surgery technique, and many institutes rely on lower levels of evidence or experts' opinions for selection of the surgery technique.

The most controversial aspect of hydatid cyst surgery is the management of residual cavity. Some surgeons prefer to leave the small to medium size cavities open without any drains, because these cysts do not harm the biliary tract and risk of infection is low and even if fluid collection occurred it can be managed using needle aspiration under ultrasonography guidance. Omentoplasty is especially popular among Turkish and Greek surgeons, in which a flap of viable omentum is placed inside the cavity resulting in obstruction of biliary fistulas. Suturing the cavity and placing drainage tubes is another method. Although it is safe and simple, patients may experience some discomfort. ${ }^{9}$ Overall we should take into consideration the location, size, and firmness of the cyst wall when deciding the appropriate surgical technique. 
Wani and colleagues assessed 50 patients undergoing liver hydatid cyst surgery using whether omentoplasty or external tube drainage methods. Their results demonstrated higher overall complications such as infection, biliary leakage, and recurrence with external tube drainage. Moreover patients with omentoplasty experienced less pain and shorter hospital stay as well as shorter time to return to normal physical activities..$^{10}$ In 2002, Mousavi and colleagues compared the external tube drainage with omentoplasty in 65 patients. ${ }^{11}$ They also reported higher post operation complications and higher length of stay in external tube drainage group; therefore they concluded that omentoplasty is superior to external tube drainage when possible. In 1998 Akin and colleagues compared different surgical techniques that use external or internal drains and surgeries not using drains and reported that using drainage systems, internal or external, results in higher complications and longer length of hospital stay; however no difference in the rate of recurrence was observed. ${ }^{12}$ Our results are in line with previous studies showing that length of hospital stay is longer in surgeries utilizing drainage systems. Moreover, the incidence of hematoma and infection was higher in patients with drains in our study; however, the difference was not statistically significant maybe due to our small sample size.

In 2007, Gourgiotis and co-workers carried out a study in England and compared different liver hydatid surgery methods in 167 patients. ${ }^{13,14}$ They showed that post-op complications were higher in patients undergoing cystostomy with capitonnage compared with patients undergoing cystostomy with omentoplasty. They reported biliary leakage as the most common complication whereas in our study no biliary leakage was observed. The reason may be that they utilized magnetic resonance imaging (MRI), which has a higher sensitivity to diagnose biliary fistula whereas we relied on ultrasonography. Moreover, higher precision in cyst cavity repair before capitonnage may result in lower biliary leakage. In contrast to their results, Manterola and colleagues published a retrospective cohort study in 2013 comparing patients undergoing omentoplasty or capitonnage for liver hydatid cysts during 2001-2008..$^{15}$ They reported that mortality rate was significantly lower in patients undergoing capitonnage after 60 months of follow-up. It seems that an increasing body of evidence is suggesting that omentoplasty tech- nique is superior to other open surgery techniques in case of post-op complications and use of draining tubes results in more complications and longer hospital stay.

Similar to our study, in most of the above mentioned studies hypertonic saline was used as a scolicidal agent. Kayaalp and others showed that scolicidal effects of hypertonic saline was limited to its low concentrations and higher concentrations resulted in complications such as peritoneal irritation (if cyst perforation occurs), sclerosing cholangitis, or biliary tract stenosis. ${ }^{16}$ Our modified technique with close drainage and repeated use of hypertonic saline can reduce the risk of spillage of daughter cysts, so without using drains we have no more complications.

\section{Limitations}

An important limitation of our study was smaller sample size in order to decrease the dispersion of data. Furthermore, due to high expenses, we could not use MRI for more accurate diagnosis of biliary fistulas and we had to rely on ultrasonography for this matter.

\section{CONCLUSION}

This method of modified surgery in hydatid cyst of the liver has good result in comparison with our traditional technique with less morbidity.

\section{ACKNOWLEDGMENT}

This paper has been extracted from the thesis of Dr. Farjad Lorestani entitled "Comparison of modified technique with routine technique in hydatid liver cyst surgery", which has been done in Endoscopic and Minimally Invasive Surgery Research Center. The authors hereby wish to thank the Research Department of Mashhad University of Medical Sciences for its support and approval of the proposal number 920431 and Mrs Sima Beigoli for her kind assistance in preparing the paper.

\section{ETHICAL APPROVAL}

There is nothing to be declared.

\section{CONFLICT OF INTEREST}

The authors declare no conflict of interest related to this work. 


\section{REFERENCES}

1. Rafiei A, Hemadi A, Maraghi S, Kaikhaei B, Craig P. Human cystic echinococcosis in nomads of southwest Islamic Republic of Iran. East Mediterr Health $J$ 2007; 13:41-8.

2. Sayek I, Tirnaksiz MB, Dogan R. Cystic hydatid disease: current trends in diagnosis and management. Surg Today 2004;34:987-96. doi:10.1007/s00595-004-2830-5.

3. Moldovan D, Moldovan CA, Ungurianu L, Ungureanu DF. A Retrospective Surgical Experience Regarding Open and Laparoscopic Procedures of the Hepatic Hydatid Cyst with an up to Date Complete Review of the Literature And a Focus On Original Romanian Techniques. J Mind Med Sci 2016;3:182-93.

4. Arif SH, Shams-Ul-Bari, Wani NA, Zargar SA, Wani MA, Tabassum R, et al. Albendazole as an adjuvant to the standard surgical management of hydatid cyst liver. Int $J$ Surg 2008;6:448-51. doi:10.1016/j.ijsu.2008.08.003.

5. Busic Z, Lemac D, Stipancic I, Busic V, Cavka M, Martic K. Surgical treatment of liver echinococcosis - the role of laparoscopy. Acta Chir Belg 2006;106:688-91. doi: 10.1080/00015458.2006.11679982.

6. Cooney RM, Flanagan KP, Zehyle E. Review of surgical management of cystic hydatid disease in a resource limited setting: Turkana, Kenya. Eur J Gastroenterol Hepatol 2004;16:1233-6.

7. Smego RA Jr, Bhatti S, Khaliq AA, Beg MA. Percutaneous aspiration-injection-reaspiration drainage plus albendazole or mebendazole for hepatic cystic echinococcosis: a meta-analysis. Clin Infect Dis 2003;37:1073-83. doi: 10.1086/378275.

8. Wang X, Li Y, Feng S. Clinical treatment of hepatic and abdominal hydatid cyst by percutaneous puncture, drainage and curettage. Zhongguo ji sheng chong xue yu ji sheng chong bing za zhi 1994;12:285-7.

9. Christians K, Pitt H. Hepatic abscess and cystic disease of the liver. Maingot's Abd Oper 2007;28:768-74.

10. Wani AA, Rashid A, Laharwal AR, Kakroo SM, Abbas M, Chalkoo MA. External tube drainage or omentoplasty in the management of residual hepatic hydatid cyst cavity: a prospective randomized controlled study. Ger Med Sci 2013;11:Doc11. doi:10.3205/000179. Print 2013.

11. Mousavi SR, Khoshnevis J, Kharazm P. Surgical treatment of hydatid cyst of the liver: drainage versus omentoplasty. Ann Hepat 2005;4:272-4.

12. Akin M, Erenoglu C, Uncu E, Basekim C, Batkin A. Surgical management of hydatid disease of the liver: a military experience. J R Army Med Corps 1998;144:139-43.

13. Marouzi S, Sharifi-rad A, Beigoli S, Teimoori Baghaee P, Assaran Darban R, Chamani J. Study on effect of lome- floxacin on human holo-transferrin in the presence of essential and nonessential amino acids: Spectroscopic and molecular modeling approaches. Int J Biol Macromol 2017;97:688-99. doi:10.1016/j.ijbiomac.2017.01.047.

14. Gourgiotis S, Stratopoulos C, Moustafellos P, Dimopoulos N, Papaxoinis G, Vougas V, et al. Surgical techniques and treatment for hepatic hydatid cysts. Surg Today 2007;37:389-95. doi:10.1007/s00595-006-3398-z.

15. Manterola C, Roa JC, Urrutia S, MINCIR Group. Treatment of the residual cavity during hepatic hydatidosis surgery: a cohort study of capitonnage vs. omentoplasty. Surg today 2013;43:1412-8. doi:10.1007/s00595-013-0570-0.

16. Kayaalp C, Balkan M, Aydin C, Ozgurtas T, Tanyuksel $\mathrm{M}$, Kirimlioglu V, et al. Hypertonic saline in hydatid disease. World J Surg 2001;25:975-9. doi:10.1007/s00268001-0065-9. 